Case Report

\title{
Polyosteotic Hydatidosis with Pathological Fracture: A Case Report
}

\author{
Rebar M. Noori Fatah* \\ Sulaimani School of Medicine, Department of Orthopedic, KRG, Iraq \\ Address correspondence to Rebar M. Noori Fatah, Sulaimani School of Medicine, Department of Orthopedic, KRG, Iraq, Tel: \\ 009647701550216; E-mail: rebarkhaffaf@gmail.com
}

Received 23 September 2017; Revised 15 May 2018; Accepted 10 October 2018

Copyright (C) 2018 Rebar M. Noori Fatah. This is an open access article distributed under the terms of the Creative Commons Attribution License, which permits unrestricted use, distribution, and reproduction in any medium, provided the original work is properly cited.

\begin{abstract}
Introduction. AHydatid disease of the bone is a rare presentation of hydatid disease since it accounts for $4 \%$ of the infection rate, which mostly involve the lungs and liver, the human being is infected with contaminated food that contain eggs and it represent the intermediate stage of the disease transmission. Case presentation. A 55 years old male who was a known case of hydatid disease of the tibia and distal femur presented with gradual onset of right hip pain which made him bed ridden, serological test proved the infection, MRI showed heavy infection of the proximal femur. Discussion. Bone involvement is very slow process owing to the compact nature, the parasites replaces the hard tissue of the bone and eventually breaches the outer cortex and spread to the soft tissue around the bone, multiple bone involvement is rare, since there are few reports of coincedence existence of pelvis and proximal femur. Skeletal hydatid disease is notorious for its high recurrence rate especially when it involve the coxofemoral and pelvic region, so it is wise to offer radical treatment from the start in a way similar to bone tumors with adjuvant chemotherapy and radiotherapy, however extensive surgeries in this region carries a high risk of complications. Conclusion. Multiple bony hydatid cyst involvement with pathological fracture is a rare presentation in the lower limb, the importance of early diagnosis is paramount to avoid major complication especially in the coxal region of the femur, skeletal survey is an important step to avoid missing skip lesions.
\end{abstract}

Keywords hydatid cyst; femur; pathological fracture

\section{Introduction}

The word Echinococcus originate from the Greek meaning "hedgehog berry" which describe the gross pathology of the lesion, the etiologic agent was first described in the 18th century, but the complete life cycle was documented in the 19th century [1], the definitive host of Echinococcus granulosus are dogs, wolves, foxes and other carnivores. This tape worm is a bout 4-6 mm in length and the infective ova shed through the feces. When swallowed by the intermediate host such as man, sheep and cattle, the embryo pass through the intestinal wall to the portal circulation where it become trapped in the liver, lungs and rarely to the other part of the body [2], regardless the location, the embryo enlarge into tinny cysts with several, completed when the definitive hosts consume the infested viscera [3].

Hydatid disease most commonly develop in the liver (50\%-77\%) and lungs $(8.5 \%-43 \%)$ [4,5], the incidence of bone infestation is about $4 \%$ mostly seen in the spine (50\%-60\%) followed by femur and tibia, humerus, skull and ribs [6].

Hydatid disease is prevalent throughout much of the world. Both forms, the alveolar and the cystic types has been reported, in Kurdistan region of Iraq the alveolar type is more common, Where the intermediate host is the cattle and sheep (15\% and 20.6\%) and in dogs (49.5\%) [7].

\section{Case report}

A 55 young male living in a district region of Sulaimani city presented with intolerable pain of the right hip of one month duration, the pain started insidiously and increased in intensity till the point he couldn't use the affected side and after a while he became bed ridden, on examination he was pale, dehydrated and emaciated, the right thigh was atrophied (mid circumference was $2 \mathrm{~cm}$ less than the left side), there was a slight swelling over the area of greater trochanter with extreme tenderness for palpation, the knee joint examination was normal, the distal pulse and nerve examination was normal, hip examination was difficult because of the pain, systemic examination otherwise was normal, his past surgical history included another surgery to his right tibia and right knee who suffered from hydatid disease of the proximal part of the 
tibia and the lateral femoral condyle which were treated by curettage and bone cement impaction 1 year ago and he received 6 months treatment of Albendazole.

Primary blood investigation was normal apart from normochromic normocytic anemia ( $\mathrm{Hb} 11 \mathrm{gm})$ and mild elevation of the ESR (50), ELISA test was strongly positive. A complete skeletal survey was performed, The plain X-rays of the pelvis showed multiple osteolytic lesions involving the intertrochanteric region and part of the neck and the head of the Femur with no evidence of periosteal reaction, Magnetic Resonance Imaging (MRI) showed a fracture line across the femoral neck with, the rest of the skeleton was uninvolved Ultrasound examination of the abdomen and pelvis otherwise was normal, pulmonary and abdominal CT scan was normal, Tru-cut biopsy proved the diagnosis, informed consent taken from the patient to replace the hip joint with the possibility of using cage for the acetabulum if it was severely involved (Figure 1).

\section{Surgical procedure}

Surgery was undertaken under spinal anesthesia, the standard Osborne posterior approach was used to expose the hip, the walls of the field was covered with packs soaked in formaldehyde $1 \%$, surprisingly the daughter cyst spell over the field from the fracture site, the bone was surprisingly weak and was easily crushed during manipulation with daughter cyst coming out from the broken crevices, after careful inspection, the proximal femur was excised with oscillating saw to the level of the lesser trochanter, we couldn't find guidelines that define the safety distance for resection of the infested bone in the literature, so it is excised the bone $2 \mathrm{~cm}$ distal to lowest point involved in the bone, later the infested bone extracted in one block, careful inspection of the acetabulum and ischium was performed through a cortical windows to exclude any possibility of involvement, a cemented cup was inserted in the acetabulum after reaming and the proximal femur was replaced by cementless tumor prosthesis, the abductor mechanism carefully attached with ethibond suture, lavage with copious amount of hypertonic normal saline done and the wound was closed over suction drain. Postoperatively the drain removed after 48 hours, partial weight bearing allowed the day after surgery using axillary crutches, Albindazole treatment started at the same time $400 \mathrm{mg}$ twice daily for 3 months. Histopathological examination of the excised fragment showed heavy infestation of the proximal femur with daughter cysts (Figure 2).

\section{Discussion}

Hydatid disease infestation of the bone happen like any part of the body by ingestion of food contaminated with eggs from carnivorous animals especially domestic dogs, in the small intestine the eggs hatch and the hexacanth embryo attach to the wall of the duodenum, later it will spread to the portal circulation and the initial spread happen [6].
One of the important feature is that bone involvement is very slow process owing to the compact nature [8], Pericyst formation does not occur as in soft tissue, this lead to aggressive proliferation in an irregular branching in the direction of least resistance, especially in the osteal canals [9], The parasites replaces the hard tissue of the bone and eventually breach the outer cortex and spread to the soft tissue around the bone [9].

Hydatid disease of bone lacks the definitive clinical manifestations, it could be presented as painless mass, neurological deficit, pathological fracture, bone sinus, sometimes it makes a diagnostic dilemma, the differential diagnosis include, chronic osteomyelitis, tuberculosis, spondylitis, bone cysts or even chondrosarcoma [4].

Most of the hydatid cyst remains silent for long period and in many occasions the infestation happen during adolescence [10,11], and the most common presentation is pathological fracture $[10,12]$ which may affect the total outcome of the process since this case is treated by tumor prosthesis with cemented acetabular cup.

Multiple bone involvement is rare, since there are few reports of co incident existence of pelvis and proximal femur. Report in the literatures about such co-incidence and if happen it is most commonly involve the spine and pelvis [13-16] which could be explained by the presence of Batson venous plexus and enormous blood supply which facilitate the process of disease spread $[14,15]$.

The current case report is about a young male farmer who had multiple hydatid cyst infection in a pattern which is unexplainable because of the wide distance between the involved regions (proximal femur, distal femur and proximal tibia).

Protection of the surgical field involve packing of the edges with scolicidal agents to prevent seeding and secondary dissemination, various agents were used including hypertonic saline, povidone idione, hydrogen peroxide, and formaldehyde (10\%), although there is no consensus exist about which solution offer protection, but most of literatures abandon the use of formaldehydes because of the risk of liver toxicity and sclerosing cholangitis whenever used intraperitoneally [16,17], chose this solution in this case because of bony location of the cyst and used it at very low concentration (1\%) instead of the standard concentration $(10 \%)$ in addition to copious irrigation with hypertonic saline after block excision $[15,16]$.

Skeletal hydatid disease is notorious for its high recurrence rate especially when it involve the coxofemoral and pelvic region $[12,17,18,19]$, there is no consensus about standard surgical procedure because of variability in the locations of the involved bone and surgical accessibility to the specific region, the estimated risk if surgery was undertaken and the degree of severity of involvement, so the spectrum may involve aggressive curettage with bone cement impaction to radical resection with wide safety 

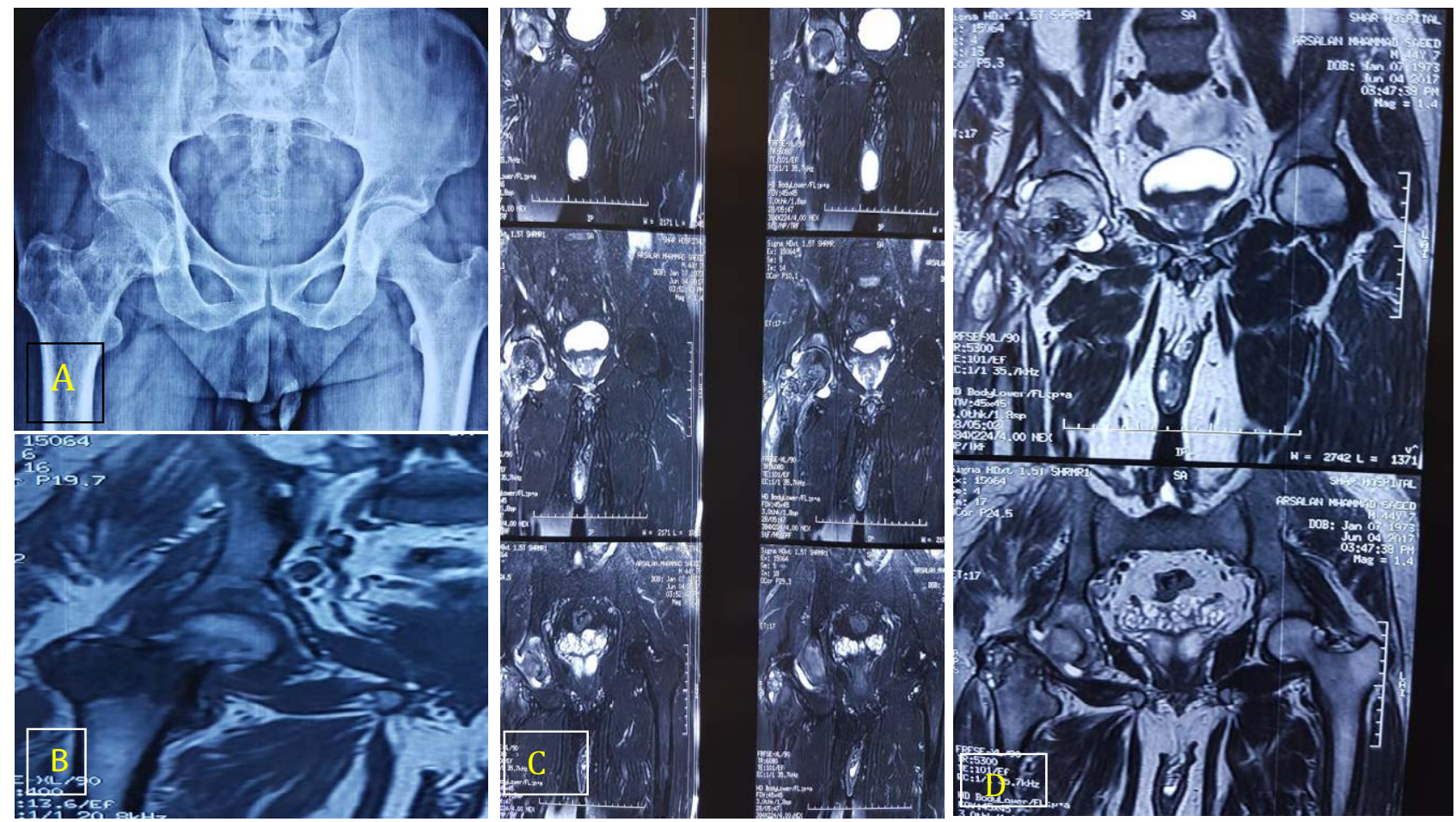

Figure 1: (a) Plain X ray of the pelvis showing haziness and moth eaten appearance of the right proximal femur (b) show the MRI coronal section on T2 show the involvement of greater trochanter(c, d) Multiple T1,T2 coronal sections showing involvement of the head with fluid inside the hip joint.
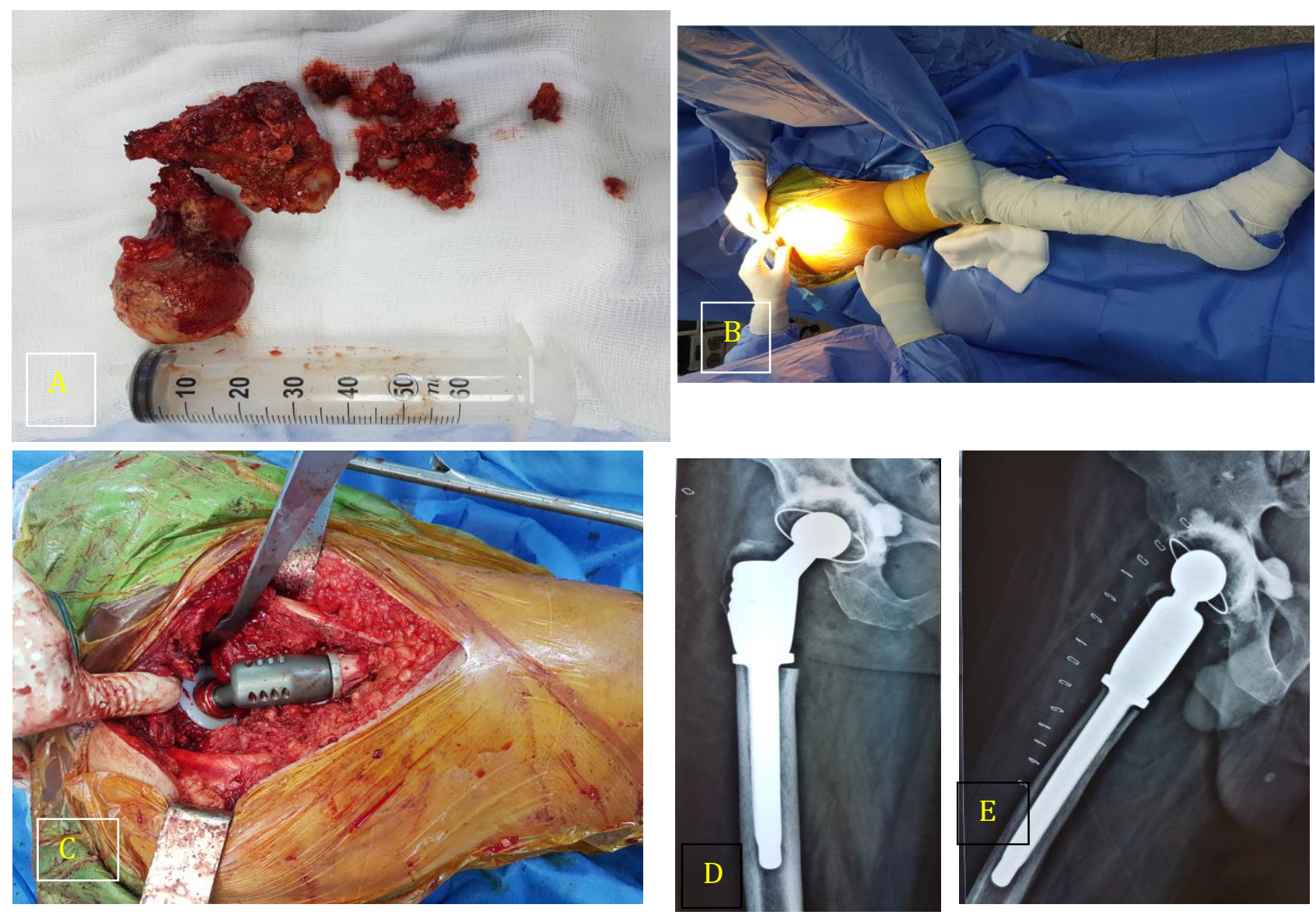

Figure 2: (a) Excised femoral head and the proximal femur you can see the fracture which run across the intertrochanteric region and the daughter cysts within the bone (b) position of the patient during posterior approach (c) the megaprosthesis with polythelene cup $(\mathrm{d}, \mathrm{e})$ the radiograph after the surgery showing well aligned components. 
margin with allograft or prosthetic reconstruction [18]. It is wiser to offer radical treatment from the start in a way similar to bone tumors with adjuvant chemotherapy and radiotherapy $[16,18]$, chemotherapy agents mainly used in the eradication of hydatid cyst is Albendazole or Albendazolum which is an antihelmenthic drug that is widely used in the treatment of various worm infestation like nematodes, giardiasis and arthrodpods in addition to its veterinary uses, its mechanism of action mainly involve degenerative changes in the intestinal wall of the worm by binding to Beta sensitive site on the tubulin thus inhibiting assembly of microtubules, in addition it impair the uptake of glucose and impair Glycogen synthesis, it is mainly given by oral administration $400 \mathrm{mg}$ twice daily with meals for 28 days in three cycles with 14 days rest between the cycles, liver function test should be checked routinely during the period of treatment because of risk of hepatotoxicity [20,21].

The aim of this report is to highlight the importance of early diagnosis before major catastrophe happen with pathological fracture especially in such critical places like the hip joint and the importance of skeletal survey in any suspected case.

\section{Conclusion}

Multiple bony hydatid cyst involvement with pathological fracture is a rare presentation in the lower limb, the importance of early diagnosis is paramount to avoid major complication especially in the coxal region of the femur, skeletal survey is an important step to avoid missing skip lesions.

\section{References}

[1] W. Joseph, Lewis Jr., Neal Koss, and D. Morris, A review of Echinocochal disease. Ann Surg, 181 (1975), 390-396.

[2] A. Herra, A. A. Mateniz, and J. Rodriguez, Spinal hydatidosis. Spine, 30 (2005), 2439-2444.

[3] G. Sapkas, D. P. Stathakopoulos, G. C. Babis, J. K. Tsarouchas, Hydatid disease of the bone and joints. 8 cases followed for 4-16 years. Acta Orthop, 69 (1998), 89-94.

[4] R. W. Charles, S. Govender, and K. S. Naidoo, Echinococcus infection of the spine with neural involvement. Spine, 13 (1988), $47-49$.

[5] C. A. Iplikcioglu, F. Kokes, A. Bayar, S. Doganay, and Z. Buharali, Spinal invasion of pulmonary hydatidosis: computed tomographic demo. Neurosurgery, 29 (1991), 467-468.

[6] X. H. Song L.W. Ding, and H. Wen, Bone hydatid disease, Postgrad Med J, 83 (2007), 536-543.

[7] S. Sadjadi, Present situation of echinococcis in the Middle East and North Africa. 55 (2006), S197-S202.

[8] R. Kumar, M. S. Cornah, and D. L. Morris, Hydatid cyst-a rare cause of pathological fracture: A case report. Injury, 15 (1984), 284-285.

[9] P. Torricelli, C. Martinelli, R. Biagini, P. Ruggieri, R. De Cristofaro, Radiographic and computed tomographic finding in hydatid disease of bone. Skeletal Radiol, 19 (1990), 435-439.

[10] A. Agulló, A. Alcalá-Santaelia, Muscle hydatidosis. About three cases. Rev Esp Reumatol, 29 (2002); 1-28.

[11] A Herra and A. Martinez, Extraspinal bone Hydatidosis. J bone Joint Surg Am, 85 (2003), 1790-1794.
[12] R. Siwach, R. Singh, V.K. Kadin, Z Singh, M. Jain, H. Madan, et al. Extensive Hydatidosis of the femur and pelvis with pathological fracture: A case report. Int J infect Dis, 13 (2009), e480-e482.

[13] R. N. Sener, C. Calli, O. Kitis, and O. Yalman, Multiple primary spinal-paraspinal hydatid cyst. Eur Radiol, 11 (2001), 2314-2316.

[14] A. Papnikolas, Osseous hydatid disease. Trans R Soc Trop Me Hyg, 102 (2008), 233-238.

[15] A. J. Alldred and N. W. Nisbet, Hydatid disease of bone in Australia. J Bone Joint Surg Surg Br, 46-B (1964), 260-267.

[16] M. Sarfaraz, I. Ansari, A. Jaiswal, and S. Sahai, Surgical management of Hydatid Cyst of liver by different scolicidal agents. World J Biol Med Sci, 2 (2015), 14-16.

[17] S. Behari, S. Banerji, R. V. Phadeke, S. Shukla, N. Krshnani, D. K. Chahabra, Multiple infected extradural parasellar hydatid cyst. Surg Neurol, 48 (1997), 3-7.

[18] A. Ibrahimi, A. Ankouz, A. Doudi, and A. Elmirini, Pelvic bone and hip joint hydatid disease revealing a retroperitoneal location. Orthopedic Review 1 (2009), e81721.

[19] E. Sozur, M. Akyuz, and S. Akbulut, Open Surgery for hepatic disease. Int Surg 99(2014), 767-769.

[20] Albendazole, The American Society of Health-System Pharmacist. Retrieved August 18, 2015 from drug.com, 2015.

[21] M. Zlitini, K. Ezzouia, H. Lebib, M. Karry, M. Kooli, and M. Mestiri, Hydatid cyst of bone: Diagnosis and treatment. World Surg, 25 (2001), 75-82. 